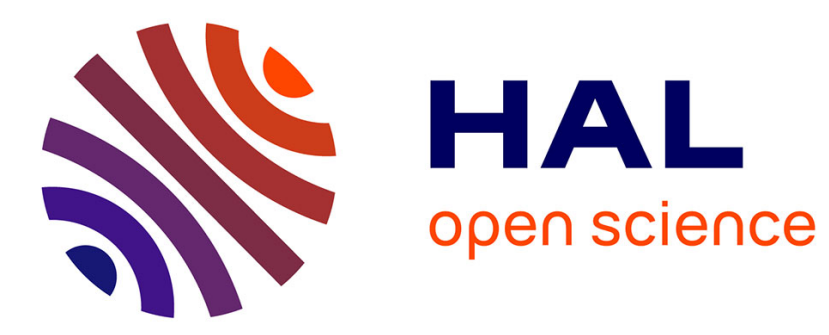

\title{
A unifying point of view on output feedback designs
}

\author{
Vincent Andrieu, Laurent Praly
}

\section{To cite this version:}

Vincent Andrieu, Laurent Praly. A unifying point of view on output feedback designs. 7th IFAC Symposium on Nonlinear Control Systems, Aug 2007, Pretoria, South Africa. pp.8-19. hal-00576953

\section{HAL Id: hal-00576953 \\ https: / hal-mines-paristech.archives-ouvertes.fr/hal-00576953}

Submitted on 15 Mar 2011

HAL is a multi-disciplinary open access archive for the deposit and dissemination of scientific research documents, whether they are published or not. The documents may come from teaching and research institutions in France or abroad, or from public or private research centers.
L'archive ouverte pluridisciplinaire HAL, est destinée au dépôt et à la diffusion de documents scientifiques de niveau recherche, publiés ou non, émanant des établissements d'enseignement et de recherche français ou étrangers, des laboratoires publics ou privés. 


\title{
A UNIFYING POINT OF VIEW ON OUTPUT FEEDBACK DESIGNS
}

\author{
V. Andrieu* $\quad$ L. Praly ** \\ * Electrical and Electronic Engineering Dept, \\ Imperial College, London, SWr $2 A Z$ \\ United Kingdom \\ vincent.andrieu@gmail.com \\ ** École des Mines de Paris \\ CAS, 35, rue Saint-Honoré, 77305 Fontainebleau Cedex \\ France \\ praly@cas.ensmp.fr
}

\begin{abstract}
:
Output feedback design for non linear systems is a difficult topic which has attracted the attention of researchers with very different approaches. We propose here a unifying point of view on possibly all these contributions.

We start with a necessary condition on the structure of the Lyapunov functions for the closed loop system. This motivates the distinction of four classes of designs which can be placed in a two by two matrix (see Table 1), with

- the direct approach, also called control error model analysis, and

- the indirect approach, also called dynamic error model analysis, as entries in one direction, and domination and cancellation as entries in the other. We show how most available results on this topic can be reinterpreted along these lines. Copyright $\bigodot^{\circ} 2007$ IFAC
\end{abstract}

Keywords: output feedback, global stabilization, non linear systems

\section{INTRODUCTION.}

\subsection{Problem statement.}

We are interested in studying the solutions which have been proposed to the following stabilization problem.

Given two continuous functions $f: \mathbb{R}^{n} \times \mathbb{R} \rightarrow \mathbb{R}^{n}$ and $h: \mathbb{R}^{n} \rightarrow \mathbb{R}$, find an integer $q$ and continuous functions $\nu: \mathbb{R}^{q} \times \mathbb{R} \rightarrow \mathbb{R}^{q}$ and $\varpi: \mathbb{R}^{q} \times \mathbb{R} \rightarrow \mathbb{R}$ such that the origin is a globally asymptotically stable equilibrium of the system:

$\dot{x}=f(x, u), y=h(x) \quad x \in \mathbb{R}^{n}, y \in \mathbb{R}$

$\dot{w}=\nu(w, y), u=\varpi(w, y) \quad w \in \mathbb{R}^{q}, u \in \mathbb{R}$, where $x$ is the state of a dynamical system to be controlled, $y$ is a measured output, $u$ is the control and $w$ is the state of a controller to be designed.

This problem is difficult since we are dealing with the global version in the general nonlinear case. It is known (Mazenc et al., 1994) that stabilizability and observability are not sufficient for the existence of a global solution, as opposed, for instance, to the semi-global case (Atassi and Khalil, 1999; Shim and Teel, 2003; Teel and Praly, 1994) or the local case, where these two properties can be taken in a very weak sense (Coron, 1994). To overcome these difficulties many different routes have been investigated by different authors and schools. Getting a complete view of all the literature is very 
difficult, because of its variety and its dispersion. In this paper we propose some guide lines to get a unifying point of view on output feedback designs with the ultimate goals of making it easier to teach this intricate topic, of getting a better grasp and allowing a better evaluation, of identifying "holes" and trying to fill them up, while finding new routes.

The paper does not contain new results, but provides new provides new ways of proving and viewing existing ones. It heavily relies on the dissertation (Andrieu, 2005). Also, it is not a survey, it simply aims at proposing a way of looking at output feedback design.

\subsection{System in normal form.}

To illustrate our presentation we shall quote known results for, but not only, systems in the so-called normal form,

$$
\begin{gathered}
\dot{z}=F\left(z, \xi_{1}\right), \\
\dot{\xi}_{1}=\xi_{2}, \ldots, \dot{\xi}_{n_{y}-1}=\xi_{n_{y}}, \\
\dot{\xi}_{n_{y}}=f\left(z, \xi_{1}, \ldots, \xi_{n_{y}}\right)+g\left(\xi_{1}\right) u \\
y=\xi_{1},
\end{gathered}
$$

for which a complete coordinate-free characterization is given in (Byrnes and Isidori, 1991, Corollary 5.7). This is one of the most general (nominal) structure for which we know how to design a globally asymptotically stabilizing output feedback and whose study has been initiated by (Kanellakopoulos et al., 1991) and (Marino and Tomei, 1991) and further developed for instance in (Andrieu and Praly, 2006; Andrieu at al, 2006; Freeman and Kokotović, 1996; Karagiannis et al., 2005; Krishnamurthy and Khorrami, 2004; Jiang et al., 2004; Marino and Tomei, 2005; Polendo and Qian, 2005; Qian and Lin, 2006) (see also the references therein).

An important point to emphasize is that, as usual with systems whose dynamic is nonlinear, the coordinates play a very significant role. By changing coordinates we may have a better view on some specificity of the system. For instance, by choosing arbitrary sufficiently smooth functions $a_{1}$ to $a_{n_{y}-1}$, positive, and $b_{1}$ to $b_{n_{y}-1}$, the above dynamic can be rewritten as:

$$
\begin{aligned}
\dot{z} & =F\left(z, y_{1}\right), \\
\dot{y}_{1} & =a_{1}\left(z, y_{1}\right) y_{2}+b_{1}\left(z, y_{1}\right), \quad y=y_{1}, \\
& \vdots \\
\dot{y}_{n_{y}-1} & =a_{n_{y}-1}\left(z, y_{1}, \ldots, y_{n_{y}-1}\right) y_{n_{y}} \\
& \quad+b_{n_{y}-1}\left(z, y_{1}, \ldots, y_{n_{y}-1}\right) \\
\dot{y}_{n_{y}} & =a_{n_{y}}\left(y_{1}\right) u+b_{n_{y}}\left(z, y_{1}, \ldots, y_{n_{y}}\right)
\end{aligned}
$$

or, in compact form, as:

$$
\dot{\chi}=A(\chi, y)+B(y) u, \quad \dot{y}=C(\chi, y),
$$

with:

$$
\chi=\left(z, y_{2}, \ldots, y_{n_{y}}\right)
$$

\subsection{An illuminating detour.}

To motivate our forthcoming classification of output feedback designs we make a detour.

Assume the origin is a globally asymptotically stable equilibrium of the interconnected system ${ }^{1}$ :

$$
\dot{\eta}_{s}=f_{s}\left(\eta_{s}, \eta_{e}\right) \quad, \quad \dot{\eta}_{e}=f_{e}\left(\eta_{s}, \eta_{e}\right)
$$

with $f_{s}$ and $f_{e}$ two continuous functions. This implies the existence of a $C^{\infty}$ positive definite and radially unbounded function $V$ whose derivative along the solutions of the system is negative definite. It follows that $\eta_{s} \mapsto \underset{\eta_{e}}{\operatorname{Argmin}} V\left(\eta_{s}, \eta_{e}\right)$ is a set valued map with non-empty values.

Lemma 1. (Prieur and Praly, 2004); (Pan et al., 2001, Section III); (Sontag, 2006, Section 13)

(1) If there exists a selection $\eta_{s} \mapsto \psi\left(\eta_{s}\right) \in$ $\underset{\eta_{e}}{\operatorname{Argmin}} V\left(\eta_{s}, \eta_{e}\right)$ which is Hölder of order strictly larger than $\frac{1}{2}$, then the following holds:

(a) $U\left(\eta_{s}\right)=V\left(\eta_{s}, \psi\left(\eta_{s}\right)\right)$ is a $C^{1}$ control Lyapunov function (CLF) for the system:

$$
\dot{\eta}_{s}=f_{s}\left(\eta_{s}, u\right)
$$

with $u=\psi\left(\eta_{s}\right)$ as a stabilizer. Precisely,

$\eta_{s} \mapsto \dot{U}_{n o m}\left(\eta_{s}\right)=\frac{\partial U}{\partial \eta_{s}}\left(\eta_{s}\right) f_{s}\left(\eta_{s}, \psi\left(\eta_{s}\right)\right)$

is a negative definite function.

(b) There exists a continuous function $H$ satisfying:

$$
\begin{aligned}
& V\left(\eta_{s}, \eta_{e}\right)=U\left(\eta_{s}\right) \\
& +\left(\eta_{e}-\psi\left(\eta_{s}\right)\right)^{T} H\left(\eta_{s}, \eta_{e}\right)\left(\eta_{e}-\psi\left(\eta_{s}\right)\right) .
\end{aligned}
$$

(2) If, for each $\eta_{s}, \frac{\partial V}{\partial \eta_{e}}\left(\eta_{s}, \eta_{e}\right)$ has a unique zero $\eta_{e}=\psi\left(\eta_{s}\right)$ and $\frac{\partial^{2} V}{\partial \eta_{e}^{2}}\left(\eta_{s}, \psi\left(\eta_{s}\right)\right)$ is positive definite then property 1a holds and there exists a change of coordinates:

$$
\left(\begin{array}{c}
\eta_{s} \\
\eta_{e}
\end{array}\right) \mapsto\left(\begin{array}{c}
\eta_{s} \\
\mathfrak{h}_{e}
\end{array}\right)=\left(\begin{array}{c}
\eta_{s} \\
\varphi\left(\eta_{s}, \eta_{e}\right)
\end{array}\right)
$$

such that we have:

$$
V\left(\eta_{s}, \eta_{e}\right)=U\left(\eta_{s}\right)+\left|\mathfrak{h}_{e}-\psi\left(\eta_{s}\right)\right|^{2} .
$$

Hence with an extra condition - Hölder selection or existence of a unique and non-degenerate critical point - global asymptotic stability of the origin

\footnotetext{
1 Index "s" is to be translated as "stabilized" and index "e" as "estimating".
} 


\begin{tabular}{c||c|c} 
& direct design $=$ estimation error model & indirect design = dynamic error model \\
\hline \hline domination & direct / domination & indirect / domination \\
\hline cancellation & direct / cancellation & indirect / cancellation
\end{tabular}

Table 1. Classification matrix

of system (8) gives rise to the decomposition (6) or (7) which exhibits:

(1) a CLF for the $\eta_{s}$ sub-system associated to the state feedback stabilizer $\psi$;

(2) a quadratic term in $\eta_{e}-\psi\left(\eta_{s}\right)$ or $\mathfrak{h}_{e}-\psi\left(\eta_{e}\right)$ that, in the present context, it is tempting to interpret as an estimation error, with $\eta_{e}$ or $\mathfrak{h}_{e}$ playing the role of an estimation of the stabilizer $\psi\left(\eta_{s}\right)$.

We have also the following decomposition for $\dot{V}$ :

$$
\begin{aligned}
& \dot{V}\left(\eta_{s}, \eta_{e}\right)=\dot{U}_{n o m}\left(\eta_{s}\right) \\
& \quad+\left(\eta_{e}-\psi\left(\eta_{s}\right)\right)^{T}\left[\mathfrak{A}\left(\eta_{s}, \eta_{e}\right) \dot{\eta}_{e}+\mathfrak{B}\left(\eta_{s}, \eta_{e}\right)\right] .
\end{aligned}
$$

Since $\dot{V}$ and $\dot{U}_{n o m}$ are negative definite, $\dot{\eta}_{e}$ must be such that the positive part of

$$
\left(\eta_{e}-\psi\left(\eta_{s}\right)\right)^{T}\left[\mathfrak{A}\left(\eta_{s}, \eta_{e}\right) \dot{\eta}_{e}+\mathfrak{B}\left(\eta_{s}, \eta_{e}\right)\right]
$$

is dominated by $\dot{U}_{n o m}\left(\eta_{s}\right)$ or simply cancelled.

The two decompositions $(6) /(7)$ and (8) are the basis of the classification we propose for output feedback designs. Specifically,

(1) when the role of $\eta_{s}$ is played by $x$ and the one of $\eta_{e}$ by $w$, i.e.:

$$
\eta_{s}=x \quad, \quad \eta_{e}=w,
$$

then we have what we call a direct design, or an estimation error model analysis.

(2) Instead, when:

$$
\begin{gathered}
\eta_{s}=w, \quad \eta_{e}=x \\
\text { or }^{2}=(w, y) \quad, \quad \eta_{e}=x(\bmod y=h(x)),
\end{gathered}
$$

then we have what we call an indirect design, or a dynamic error model analysis.

Each of these two classes is subdivided into two sub-classes, depending on whether the choice of $\dot{\eta}_{e}$ is made to cancel or dominate of the bad terms, as discussed above.

In summary, Lemma 1 is the basis for the classification in Table 1. Such a classification and the terminology we are using are not new. They are borrowed from the literature on adaptive linear control (see (Ioannou and Sun, 1996)) and have been used in the non-linear context in (Pomet, 1989).

${ }^{2} x(\bmod y=h(x))$ means that $\eta_{e}$ is made of the components of $x$ which are not directly given by the knowledge of $y=h(x)$.

\section{DIRECT DESIGN = ESTIMATION ERROR MODEL ANALYSIS.}

\subsection{The context.}

Let system (5) represent system (1) with the identification:

$$
\eta_{s}=x \quad, \quad \eta_{e}=w .
$$

Lemma 1 says (ignoring the extra condition!) that, if the stabilization problem is solved, then there exist a function $\psi$ and a Lyapunov function $V$ such that we have:

$V(x, w)=U(x)+(w-\psi(x))^{T} H(x, w)(w-\psi(x))$ $\dot{V}(x, w)=\dot{U}_{n o m}(x)$

$$
\begin{array}{ll} 
& +(w-\psi(x))^{T}[\mathfrak{A}(x, w) \dot{w}+\mathfrak{B}(x, w)] \\
<0 \quad \forall(x, w) \neq 0
\end{array}
$$

$\dot{U}_{n o m}(x)=\frac{\partial U}{\partial x}(x) f(x, \varpi(\psi(x), h(x)))<0 \quad \forall x \neq 0$

These three equations can be interpreted as follows.

(1) As mentioned above, we can view $w$ as an estimator of $\psi(x)$, a state feedback stabilizer for the system to be controlled.

(2) The third equation says that, for the system:

$$
\dot{x}=f(x, u),
$$

we have a CLF $U$ to which is associated the state feedback:

$$
u=\phi(x)=\varpi(\psi(x), h(x)) .
$$

Embedded here is a control reparameterization $(v, y) \mapsto u$ allowing us to go from the estimated $\psi$ to the state feedback $\phi$. Typical such reparameterizations are:

$$
u=\varpi(v, y) \quad \text { or } \quad u=\varpi(\psi(v), y) .
$$

In the former case, $w$ should be an estimation of $\psi(x)$, a function of the state of the system to be controlled. In the latter, $w$ should be an estimation of the state $x$ itself.

(3) Finally, the second equation says that either $\dot{w}$ is supposed to be such that we get:

$$
(w-\psi(x))^{T}[\mathfrak{A}(x, w) \dot{w}+\mathfrak{B}(x, w)] \leq 0 ;
$$

this is what we regard as "cancellation". Alternatively we can take advantage of the negativeness of $\dot{U}_{n o m}(x)$ and simply guarantee that $(w-\psi(x))^{T}[\mathfrak{A}(x, w) \dot{w}+\mathfrak{B}(x, w)]$ is dominated by $\dot{U}_{\text {nom }}(x)$; this is "domination". 
We call this approach direct design since $w$ is estimating $\psi(x)$, the reparameterized state feedback, which is the only information we need for the stabilization of (9). But $w$ is only an estimation, hence, when implementing the control as (see (1)):

$$
u=\varpi(w, y)=\varpi(w, h(x)),
$$

we are introducing the estimation error $\psi(x)-w$ as a disturbance.

According to this direct approach, an output feedback design consists in the following steps:

(1) Design a state feedback $\phi(x)$ for system (9),

(2) Do a control reparameterization of this state feedback as:

$$
\phi(x)=\varpi(\psi(x), h(x)),
$$

(3) Design an observer for the reparameterized control law $\psi(x)$ which also fulfils the domination or cancellation task.

We can re-interpret along these lines what is proposed for instance in (Arcak and Kokotović, 2001-TAC; Polendo and Qian, 2005; Qian and Lin, 2006), and (Andrieu and Praly, 2006, Section 2).

Example 1 (Kokotović, 1992): Consider the system:

$\dot{x}_{1}=-x_{1}+\left(u-x_{2}\right) x_{1}^{2}, \dot{x}_{2}=-x_{2}+x_{1}^{2}, y=x_{1}$.

A CLF and an associated stabilizer are:

$U\left(x_{1}, x_{2}\right)=x_{1}^{4}+x_{2}^{2} \quad, \quad \phi\left(x_{1}, x_{2}\right)=x_{2}$.

If we proceed with no control reparameterization, i.e. we let:

$$
\psi\left(x_{1}, x_{2}\right)=\phi\left(x_{1}, x_{2}\right)=x_{2},
$$

we are left with designing an observer for $x_{2}$. This task is easily fulfilled by:

$$
\dot{\hat{x}}_{2}=-\hat{x}_{2}+y^{2}, \quad w=\hat{x}_{2},
$$

yielding:

$$
\overparen{\left(x_{2}-\hat{x}_{2}\right)^{2}}=-2\left(x_{2}-\hat{x}_{2}\right)^{2} .
$$

So we are done ... Unfortunately no, some solution of the closed loop system escapes to infinity in finite time! The problem is that, in this design, we have skipped the step of "cancellation" or "domination" mentioned above. In other words we do not have taken care of the error between the (reparameterized) stabilizer and its estimation (see (Praly and Arcak, 2004; Arcak, 2005)).

\subsection{Domination.}

Example 1 continued: Consider again system (10) and proceed with the control reparameterization $(v, y) \mapsto u=v-a(y)$, where the function $a$ is a degree of freedom to be exploited for domination. With (11), this implies that the term $\psi\left(x_{1}, x_{2}\right)$ to be estimated by $w$ is:

$$
\psi\left(x_{1}, x_{2}\right)=x_{2}+a\left(x_{1}\right) .
$$

On the other hand, for the CLF $U$ in (11), we have:

$\dot{U}\left(x_{1}, x_{2}\right) \leq-U\left(x_{1}, x_{2}\right)+4 x_{1}^{5}\left(v-x_{2}-a\left(x_{1}\right)\right)$

Hence, when implementing the output feedback as $v=w$, we introduce the disturbance:

$$
x_{1}^{5}\left(w-\psi\left(x_{1}, x_{2}\right)\right)=x_{1}^{5}\left(w-x_{2}-a\left(x_{1}\right)\right) .
$$

By inequality (13), this term is not a worry if it is integrable when evaluated along the solutions of the closed loop system. Hence we are led to say that $w$ is a good estimate of $\psi\left(x_{1}, x_{2}\right)=x_{2}+a\left(x_{1}\right)$ if this integrability property is satisfied. Motivated by the identity:

$\overbrace{x_{2}+a\left(x_{1}\right)}=-x_{2}+x_{1}^{2}+a^{\prime}\left(x_{1}\right)\left[-x_{1}+\left(u-x_{2}\right) x_{1}^{2}\right]$, we propose to estimate $\psi\left(x_{1}, x_{2}\right)$ by $w$ given by: $\dot{w}=x_{1}^{2}+a^{\prime}\left(x_{1}\right)\left[-x_{1}+u x_{1}^{2}\right]-b\left(x_{1}\right)\left[w-a\left(x_{1}\right)\right]$ with $b$ another degree of freedom. The integrability property is satisfied when we choose, for instance, $a$ and $b$ as:

$$
a\left(x_{1}\right)=\frac{x_{1}\left|x_{1}\right|^{3}}{4} \quad, \quad b\left(x_{1}\right)=1+\left|x_{1}\right|^{5}
$$

since we have in this case:

$\overparen{\left|w-x_{2}-a\left(x_{1}\right)\right|} \leq-\left(1+\left|x_{1}\right|^{5}\right)\left|w-x_{2}-a\left(x_{1}\right)\right|$.

2.2.1. Design via ISS or iISS domination.

What has been done in the above example can be formalized in general. The idea is to exploit the possibility that, maybe after a control reparameterization, we could find a state feedback making the system input-to-state stable (ISS) or integral input-to-state stable (iISS) with respect to an input disturbance (see (Andrieu et al, 2006; Freeman and Kokotović, 1993; Sontag, 1990)).

Proposition 1. (ISS or iISS domination). The output feedback stabilization problem is solved if the integer $q$ and the continuous functions $\nu: \mathbb{R}^{q} \times$ $\mathbb{R} \rightarrow \mathbb{R}^{q}$ and $\varpi: \mathbb{R}^{q} \times \mathbb{R} \rightarrow \mathbb{R}$ are such that the following holds:

(1) There exist a control reparameterization $u=$ $\varpi(v, y)$ and a corresponding state feedback $\psi$ making the system:

$$
\dot{x}=f(x, \varpi(\psi(x)+e, h(x)))
$$

$(\gamma)$-iISS (respectively ISS), i.e. there exist a Lyapunov function $U$ and a continuous function $\gamma$, zero at zero, satisfying:

$$
\dot{U}(x) \leq \dot{U}_{n o m}(x)+\gamma(|e|) \quad \forall(x, e),
$$
with $\dot{U}_{\text {nom }}$ negative definite (respectively, and radially unbounded);

(2) The state $w$ of: 


$$
\dot{w}=\nu(w, y)
$$

is an estimate of $\psi(x)$ such that $\gamma(\mid w-$ $\psi(x) \mid$ ) is integrable (respectively, bounded and converges to 0 ) along any solution of the closed loop system.

A straightforward application of this design via ISS domination yields the following result for systems in normal form (2).

Proposition 2. (Andrieu and Praly, 2006) If:

(1) the subsystem $\dot{z}=F\left(z, y_{1}\right)$ is linear in $y_{1}$ and feedback linearizable;

(2) there exist functions $a_{1}$ to $a_{n_{y}-1}$, positive, and $b_{1}$ to $b_{n_{y}-1}$, a continuous function $y \mapsto$ $K(y)$ and a positive definite symmetric matrix $P$ satisfying, for all $(\chi, y)$ (see notation in $(3))^{3}$,

$P \frac{\partial(A-K C)}{\partial \chi}(\chi, y)+\frac{\partial(A-K C)}{\partial \chi}(\chi, y)^{T} P<0$; then we can solve the output feedback stabilization problem for system (2).

The first condition guarantees the existence of a continuous function $\phi$ such that the system (see $(3))$ :

$\dot{\chi}=A(\chi, y)+B(y) \phi(\chi+e, y), \quad \dot{y}=C(\chi, y)$ is ISS with $e$ as input. This has been established in (Freeman and Kokotović, 1993). The second condition guarantees that, by selecting:

$$
\begin{aligned}
\dot{w}=A\left(w+\int_{0}^{y} K(s) d s, y\right) & +B(y) u \\
& -K(y) C\left(w+\int_{0}^{y} K(s) d s, y\right),
\end{aligned}
$$

we get that $e=w+\int_{0}^{y} K(s) d s-\chi$ is bounded and converges to 0 along any solution. Hence a direct design via ISS domination (Proposition 1) can be done with the control reparameterization:

$$
u=\varpi(v, y)=\phi\left(v+\int_{0}^{y} K(s) d s, y\right)
$$

and:

$$
\psi\left(\chi, y_{1}\right)=\chi-\int_{0}^{y_{1}} K(s) d s
$$

Proposition 2 relies on ISS domination. To illustrate iISS domination, consider the system in normal form:

$$
\dot{\xi}_{1}=\xi_{2}, \quad \dot{\xi}_{2}=\xi_{2}^{2}+u, \quad y=\xi_{1} .
$$

Let:

$$
U\left(\xi_{1}, \xi_{2}\right)=\xi_{1}^{2}+\xi_{2}^{2} \exp \left(-2 \xi_{1}\right) .
$$

We have:

$$
\dot{U}\left(\xi_{1}, \xi_{2}\right)=2 \xi_{2}\left(\xi_{1}+u \exp \left(-2 \xi_{1}\right)\right) .
$$

Hence a stabilizing state feedback is:

\footnotetext{
3 Sufficient conditions for this assumption to hold can be found for instance in (Arcak and Kokotović, 2001AUT; Krishnamurthy et al., 2002).
}

$$
\phi\left(\xi_{1}, \xi_{2}\right)=-\xi_{1} \exp \left(2 \xi_{1}\right)-\xi_{2} .
$$

Consider now the control reparameterization:

$\left(v, \xi_{1}\right) \mapsto u=v \exp \left(\xi_{1}\right)-\xi_{1} \exp \left(2 \xi_{1}\right)+1-\exp \left(\xi_{1}\right)$. and the corresponding (new) state feedback:

$$
\psi\left(\xi_{1}, \xi_{2}\right)=-\xi_{2} \exp \left(-\xi_{1}\right)-\exp \left(-\xi_{1}\right)+1 .
$$

Observe that this makes the system $L^{2}$-ISS since:

$$
\begin{aligned}
u=\left[\psi\left(\xi_{1}, \xi_{2}\right)+e\right] & \exp \left(\xi_{1}\right) \\
& -\xi_{1} \exp \left(2 \xi_{1}\right)+1-\exp \left(\xi_{1}\right)
\end{aligned}
$$

is such that we obtain:

$$
\dot{U}\left(\xi_{1}, \xi_{2}\right) \leq-\xi_{2}^{2} \exp \left(-2 \xi_{1}\right)+e^{2} .
$$

Actually this is not the $L^{2}$-ISS property stricto sensu since $\left(\xi_{1}, \xi_{2}\right) \mapsto \xi_{2}^{2} \exp \left(-2 \xi_{1}\right)$ is not a negative definite function. Nevertheless we go one and observe that the function $\gamma$ in the iISS domination design (see Proposition 1) is:

$$
\gamma(s)=s^{2} .
$$

To satisfy the second assumption of Proposition 1 , we differentiate $\psi\left(\xi_{1}, \xi_{2}\right)$ along the solutions to obtain:

$$
\dot{\psi}=-\exp (-y) u-\psi-\exp (-y)+1 .
$$

Hence, to estimate $\psi\left(\xi_{1}, \xi_{2}\right)$ by $w$, we propose:

$$
\dot{w}=-\exp (-y) u-w-\exp (-y)+1 \text {. }
$$

This is satisfactory since we get:

$\widetilde{\left(w-\psi\left(\xi_{1}, \xi_{2}\right)\right)^{2}}=-2\left(w-\psi\left(\xi_{1}, \xi_{2}\right)\right)^{2}=-2 \gamma(e)$.

It can be checked, by means of an invariance principle, that we do have a solution for the output feedback stabilization problem.

\subsubsection{Design from the natural stability margin.}

It is not always possible to render a system ISS or iISS with respect to an input disturbance (see (Freeman, 1995; Chung, 1999)). In general, we only have that, for given CLF $U$, reparameterization $\varpi$ and state feedback $\psi$, there exists a positive definite function $\rho$ - the stability margin - such that we have:

$$
\begin{aligned}
& \frac{\partial U}{\partial x}(x) f(x, \varpi(\psi(x)+e, h(x)))<0 \\
& \forall(e, x):|e|<\rho(|x|) .
\end{aligned}
$$

In this case, the estimation of $\psi(x)$ by $w$ must be done to match the inequality $|w-\psi(x)|<\rho(x)$ along the solutions as quickly as possible and before any possible finite escape time. This estimation task is more demanding than the one involved in the ISS or iISS domination design. It is possible for instance if: a. $\psi(x)$ is uniformly completely observable ${ }^{4}$;

\footnotetext{
4 Uniform complete observability: There exists a $C^{1}$ function $\Phi$ and an integer $m$ such that, for any solution $t \mapsto$ $\left(x(t), u_{0}(t), \ldots, u_{m-1}(t)\right)$, maximally defined on $\left(T_{-}, T_{+}\right)$, of:

$\dot{x}=f\left(x, u_{0}\right), \quad \dot{u}_{0}=u_{1}, \ldots, \dot{u}_{m-1}=u_{m}, y=h(x)$,
} 
b. a bound on the norm of $x$ can be estimated.

In this case, it is sufficient to use a "high gain observer" with a dynamical gain tuned from this bound.

Condition b. is met, for instance, if we restrict our interest to semi-global stabilization, as established in (Atassi and Khalil, 1999; Teel and Praly, 1994). In this case the bound is obtained from knowing that the initial conditions are in a given compact set.

It is also met in the global case studied in (Praly and Astolfi, 2005) where the bound is obtained from a state norm estimator whose existence follows, for instance, from an Input-Output to State Stability property for system (9). Precisely, the following holds.

Proposition 3. (Praly and Astolfi, 2005). If system (9) is stabilizable, uniformly completely observable and state norm detectable ${ }^{5}$ then we can solve the output feedback stabilization problem.

The above conditions a. and b. can be relaxed, at least at a conceptual level, as follows:

Unboundedness Observability: If the state escapes in finite time to infinity so does the output or the input.

Small time distinguishability: For some function $t \mapsto u^{*}(t)$ and for all $T>0$, the function $x \mapsto\{t \in[0, T) \mapsto y(t, x)\}$ is injective.

See (Shim and Teel, 2003) for the semi-global case and (Mazenc and Praly, 1993) for the global case. See also (Coron, 1994).

\subsection{Cancellation.}

A direct design via cancellation follows from a straightforward application of what we learned in

we have, for each $t$ in $\left(T_{-}, T_{+}\right)$,

$$
\psi(x(t))=\Phi\left(y(t), y_{1}(t), \ldots, y_{m}(t), u_{0}(t), \ldots, u_{m-1}(t)\right),
$$

where $y_{i}$ denotes the $i$ th time derivative of the output $y$.

5 State norm detectability: There exist $C^{1}$ functions $W, \alpha$ and $\beta$, such that $\alpha$ is non-increasing in its first argument, $\beta$ is non-decreasing in its first argument and we have:

$$
\begin{aligned}
& \dot{W}(x)=\frac{\partial W}{\partial x}(x) f(x, u) \leq \alpha(W(x), u, h(x)) \quad \forall(x, u) \\
& |x| \leq \beta(W(x), h(x)) \quad \forall x, \quad \alpha(0, u, h) \geq 0 \quad \forall(u, h) .
\end{aligned}
$$

Moreover there exist a continuous function $\bar{\alpha}$, two nonnegative real numbers $c_{1}$ and $W_{*}$ and four strictly positive real numbers $c_{2}, c_{3}, \sigma$ and $\alpha_{*}$ satisfying:

$$
\begin{array}{r}
\alpha\left(\left(1+c_{3}\right) W+c_{1}, u, h\right)+c_{2} \leq\left[1+c_{3}\right] \alpha(W, u, h) \\
\alpha(W, u, h) \leq \bar{\alpha}(u, h), \quad \forall(W, u, h) \\
\alpha(W, u, h) \leq-\alpha_{*} \quad \forall(W, u, h): W \geq W_{*},|u|+|h| \leq \sigma .
\end{array}
$$

Lemma 1. As an illustration, consider system (14). A (weak) CLF is given by (15). Therefore, by (7), consider the function:

$V\left(\xi_{1}, \xi_{2}, w\right)=\xi_{1}^{2}+\xi_{2}^{2} \exp \left(-2 \xi_{1}\right)+\left(w-\psi\left(\xi_{1}, \xi_{2}\right)\right)^{2}$. Our objective is to define $\dot{w}$ and the function $\psi$ so that the derivative of $V$ along the closed loop solutions is non-positive. Note that we have:

$$
\begin{aligned}
& \dot{V}\left(\xi_{1}, \xi_{2}, w\right)= \\
& 2 \xi_{2}[y+u \exp (-2 y)]+2[w-\psi][\dot{w}-\dot{\psi}]
\end{aligned}
$$

This derivative is non-positive if, for instance, we have:

$\left(\begin{array}{c}y+u \exp (-2 y) \\ \dot{w}-\dot{\psi}\end{array}\right)=-M\left(y, \xi_{2}, w\right)\left(\begin{array}{c}\xi_{2} \\ w-\psi\left(y, \xi_{2}\right)\end{array}\right)$

where $M$ is any matrix with non-negative symmetric part. The difficulty is that the solution $(u, \dot{w})$ of this equation cannot depend on $\xi_{2}$. One way to satisfy this constraint is to choose:

$$
\psi\left(\xi_{1}, \xi_{2}\right)=\xi_{1}, \quad M\left(y, \xi_{2}, w\right)=\left(\begin{array}{rr}
0 & -1 \\
1 & 1
\end{array}\right) .
$$

This yields the output feedback:

$$
u=(w-2 y) \exp (2 y), \quad \dot{w}=-(w-y) .
$$

It can be checked, by means of an invariance principle, that it does solve the output feedback stabilization problem.

More generally, when system (9) is affine in the control, i.e. we have:

$$
\dot{x}=f(x)+g(x) u,
$$

a direct design via cancellation can be performed by introducing the function:

$$
V(x, w)=U(x)+|\mathfrak{h}(w, x)-\psi(x)|^{2},
$$

where the functions $U$ and $\mathfrak{h}$ are to be defined so that $U$ is a CLF for (16), with associated state feedback $\phi(x)$, and $w \mapsto \mathfrak{h}(w, x)$ is a diffeomorphism for all $x$. The derivative is:

$$
\begin{aligned}
\dot{V}(x, w)= & \dot{U}_{\text {nom }}(x)+L_{g} U(x)[u-\phi(x)] \\
+ & {[\mathfrak{h}(w, x)-\psi(x)]^{T} \times } \\
& \times\left[\frac{\partial \mathfrak{h}}{\partial w}(w, x) \dot{w}+\frac{\partial \mathfrak{h}}{\partial x}(w, x) \dot{x}-\dot{\psi}(x)\right] .
\end{aligned}
$$

It is non-positive if we have:

$$
\begin{aligned}
& \left(\begin{array}{c}
u-\phi(x) \\
\frac{\partial \mathfrak{h}}{\partial w}(w, x) \dot{w}+\frac{\partial \mathfrak{h}}{\partial x}(w, x) \dot{x}-\dot{\psi}(x)
\end{array}\right) \\
& =-M(x, w)\left(\begin{array}{c}
L_{g} U(x) \\
\mathfrak{h}(w, x)-\psi(x)
\end{array}\right),
\end{aligned}
$$

where $M$ is any matrix with non-negative symmetric part. To complete the design it remains to select the dimension $q$ of $w$, the functions $\mathfrak{h}$ and $\psi$, and the matrix $M$ so that the solution $(u, \dot{w})$ of equation (17) depends only on $y=h(x)$ and $w$. In general such a selection is very difficult to make and may be even impossible if $U$ and $\phi$ are not appropriately selected. Some conditions 
under which it can be done are given in (Prieur and Praly, 2004). They are satisfied for instance by passive systems whose output is the derivative of the measurement $y$ (see (Ailon and Ortega, 1993; Ortega et al., 1995)). See also (Pomet et al., 1993).

\section{INDIRECT DESIGN = DYNAMIC ERROR MODEL ANALYSIS.}

\subsection{The context.}

To introduce in a simple way the indirect design based on dynamic error model analysis, we assume that the output can be taken as one coordinate. This means that $x$ can be decomposed as $x=$ $(\chi, y)$ and the dynamic is:

$$
\dot{\chi}=A(\chi, y, u) \quad, \quad \dot{y}=C(\chi, y, u) .
$$

Then, let system (5) represent system (1) with the identification:

$\eta_{s}=(w, y) \quad, \quad \eta_{e}=\chi \quad(=x(\bmod y=h(x)))$.

Lemma 1 says (ignoring again the extra condition) that, if the stabilization problem is solved then there exists a Lyapunov function $V$ admitting the decomposition:

$$
\begin{aligned}
& V(\chi,(w, y))=U(w, y) \\
& \quad+(\chi-\psi(w, y))^{T} H(\chi,(w, y))(\chi-\psi(w, y)) .
\end{aligned}
$$

We now study the meaning of the two terms on the right hand side.

(1) We have seen in Section 1.3 that $\chi-\psi(w, y)$ should be seen as an estimation error. This leads us to interpret the equations:

$$
\dot{w}=\nu(w, y), \quad \hat{\chi}=\psi(w, y),
$$

as those of an observer of the unmeasured state components $\chi$.

(2) Again, according to Section 1.3, $U$ is a CLF for the $\eta_{s}=(w, y)$-subsystem which is:

$$
\dot{w}=\nu(w, y), \quad \dot{y}=C(\chi, y, \varpi(w, y)),
$$

with $\chi$ as control and $\chi=\psi(w, y)$ is a stabilizing state feedback.

To get a better grasp on this interpretation it is informative to rewrite (19) and (20) as:

$$
\begin{aligned}
\dot{w} & =\nu(w, y), \\
\dot{y} & =C(\hat{\chi}, y, \varpi(w, y))+d_{y}(\chi, w, y), \\
\hat{\chi} & =\psi(w, y) .
\end{aligned}
$$

This shows a nominal system with $\hat{\chi}$ as both input and output, and with:

$d_{y}(\chi, w, y)=C(\chi, y, \varpi(w, y))-C(\hat{\chi}, y, \varpi(w, y))$ as disturbance. The presence of $d_{y}$ explains why we call (20) a model with an error in its dynamic or shortly dynamic error model. Then, as $U$ is a CLF for the nominal system, with $\hat{\chi}=\psi(w, y)$ the associated stabilizing state feedback, we see that one task in designing $\nu, \varpi$ and indirectly $\psi$ is to achieve stabilization in spite of the presence of $d_{y}$. On the other hand this disturbance is necessary for guaranteeing the convergence of $\hat{\chi}$ towards $\chi$ which is needed to transfer the stabilization property obtained for $(w, y)$ to $\chi$. It is because stabilization for $\chi$ is obtained in this indirect way that we call this design indirect.

Furthermore, we remark that, if (19) is indeed an observer of $\chi$, then we should have $\{(\chi, y, w)$ : $\chi=\psi(w, y)\}$ as an invariant manifold for (18). This yields the identity:

$$
\begin{aligned}
A(\psi(w, y), y, \varpi(w, y))=\frac{\partial \psi}{\partial w}(w, y) \nu(w, y) & \\
+ & \frac{\partial \psi}{\partial y}(w, y) C(\psi(w, y), y, \varpi(w, y))
\end{aligned}
$$

and also, by differentiating $\hat{\chi}=\psi(w, y)$,

$$
\begin{aligned}
& \dot{y}=C(\hat{\chi}, y, \varpi(w, y))+d_{y}(\chi, w, y), \\
& \dot{\hat{\chi}}=A(\hat{\chi}, y, \varpi(w, y))+\frac{\partial \psi}{\partial y}(w, y) d_{y}(\chi, w, y) .
\end{aligned}
$$

Therefore an output feedback design according to this indirect approach consists in the following steps:

(1) Design an observer for the state unmeasured part $\chi$, i.e. a correction term $\left(d_{y}, \psi\right)$, with the objective that any good property (e.g. convergence) obtained for $\hat{\chi}$ is transferred to $\chi$

(2) Design a control law $\varpi$ ensuring good properties for $\hat{\chi}$ in spite of the presence of the correction term.

Most of the publications on global stabilization by output feedback can be re-interpreted along these lines. In particular this is the case of (Andrieu and Praly, 2006; Arcak, 2005; Kanellakopoulos et al., 1991; Krishnamurthy and Khorrami, 2004; Marino and Tomei, 1991; Jiang et al., 2004; Marino and Tomei, 2005; Praly and Arcak, 2004)

\subsection{Domination.}

Example 1 continued: To illustrate how the indirect approach works with a domination design, we use the system in Example 1. As already seen, a simple observer for $x_{2}$ is obtained by simply copying the system, i.e.:

$$
\dot{\hat{x}}_{2}=-\hat{x}_{2}+y^{2} \text {. }
$$

This gives an error $e_{2}=\hat{x}_{2}-x_{2}$ which is exponentially decaying along any solution. In this case the system (21) takes the form:

$$
\dot{y}=-y+\left(u-\hat{x}_{2}\right) y^{2}+y^{2} e_{2}, \quad \dot{\hat{x}}_{2}=-\hat{x}_{2}+y^{2} .
$$

For such a system, we observe that the control

$$
u=\varpi\left(y, \hat{x}_{2}\right)=\hat{x}_{2}-y
$$


guarantees boundedness and convergence to 0 of any solution whatever the input $t \mapsto e_{2}(t)$ is as long as it is a continuous bounded function which converges to 0 as $t$ goes to $+\infty$. Actually it can be established that the stabilization problem is solved by using the Lyapunov function $y^{2}+e_{2}^{2}$. An important point to notice is the presence of $-y$ in the expression of $\varpi$. It was not present in (12).

\subsubsection{Design via ISS or iISS domination.}

What has been done in the above example can be formalized as follows.

Proposition 4. (ISS or iISS domination). The output feedback stabilization problem is solved if we can find three functions $k_{l}, k_{r}$ and $\varpi$ such that

(1) the system:

$$
\dot{\hat{x}}=f(\hat{x}, \varpi(\hat{x}, y))+k_{l}(\hat{x}, y) d
$$

is $(\gamma)$ iISS (resp. ISS);

(2) along the solutions of:

$$
\begin{gathered}
\dot{x}=f(x, \varpi(\hat{x}, y)), \\
\dot{\hat{x}}=f(\hat{x}, \varpi(\hat{x}, y))+k_{l}(\hat{x}, y) k_{r}(\hat{x}, y), \\
\gamma\left(\left|k_{r}(\hat{x}, y)\right|\right) \text { is integrable (resp. bounded and } \\
\hat{x}-x \text { converges to } 0) .
\end{gathered}
$$

In the context of this proposition, we have $q=n$ and

$$
\nu(w, y)=f(w, \varpi(w, y))+k_{l}(w, y) k_{r}(w, y) .
$$

For example, a straightforward application of this design via iISS domination yields the following result for systems in normal form (2).

\section{Proposition 5. (Andrieu and Praly, 2006) If:}

(1) there exist functions $a_{1}$ to $a_{n_{y}-1}$, positive, and $b_{1}$ to $b_{n_{y}-1}$, a continuous function $y \mapsto$ $K(y)$ and a positive definite symmetric matrix $P$ satisfying, for all $(\chi, y)$ (see notation in $(3))$,

$$
\begin{gathered}
P \frac{\partial(A-K C)}{\partial \chi}(\chi, y)+\frac{\partial(A-K C)}{\partial \chi}(\chi, y)^{T} P \\
<-\frac{\partial C}{\partial \chi}(\chi, y)^{T} \frac{\partial C}{\partial \chi}(\chi, y) ;
\end{gathered}
$$

(2) there exists a sufficiently many times differentiable function $\phi_{z}$ such that the system:

$$
\dot{z}=F\left(z, \phi_{z}(z)\right)+K_{z}(y) d
$$

is $L^{2}$-ISS, with $K_{z}$, the $z$-component of $K$ above,

then we can solve the output feedback stabilization problem for system (2).

The first condition guarantees that

$$
d_{y}=C(\chi, y)-C(\hat{\chi}, y)
$$

is square integrable along any solution of the system

$$
\begin{aligned}
& \dot{\chi}=A(\chi, y)+B(y) u, \quad \dot{y}=C(\chi, y), \\
& \dot{\hat{\chi}}=A(\hat{\chi}, y)+B(y) u+K(y)[C(\chi, y)-C(\hat{\chi}, y)]
\end{aligned}
$$

and for any input $t \mapsto u(t)$. The second condition guarantees the existence of a continuous function $\phi$ such that the system:

$$
\begin{aligned}
& \dot{\chi}=A(\chi, y)+B(y) \phi(\chi, y)+K(y) d_{y}, \\
& \dot{y}=C(\chi, y)+d_{y},
\end{aligned}
$$

is $L^{2}$-ISS with $d_{y}$ as input. This has been established in (Kanellakopoulos et al., 1991).

Proposition 5 follows the route of $L^{2}$-ISS domination. We provide now an illustration of $L^{1}$-ISS domination. Consider the system in normal form:

$$
\dot{z}=3 z+2 z^{3}+y, \quad \dot{y}=z+z^{3}+u .
$$

An observer for $z$ is given by:

$$
\dot{w}=-\hat{z}-2 \hat{z}^{3}+y-4 u, \hat{z}=w+4 y .
$$

It yields the disturbance

$$
d_{y}=\left[z+z^{3}\right]-\left[\hat{z}+\hat{z}^{3}\right],
$$

which is $L^{1}$ integrable along any solution ${ }^{6}$. Then we see that, by selecting:

$$
\phi_{z}(z)=-4 z-3 z^{3}, \quad U(z)=\sqrt{1+z^{2}}-1,
$$

gives:

$\frac{d U}{d z}(z)\left[3 z+2 z^{3}+\phi_{z}(z)+d\right] \leq-z^{2} \sqrt{1+z^{2}}+|d|$.

It follows from (Andrieu and Praly, 2006), that there exists a continuous function $\phi$ such that the system:

$\dot{z}=3 z+2 z^{3}+y+4 d_{y}, \quad \dot{y}=z+z^{3}+\phi(z, y)+d_{y}$ is $L^{1}$-ISS with $d_{y}$ as input. Hence the output feedback:

$\dot{w}=-\hat{z}-2 \hat{z}^{3}+y-4 u, \hat{z}=w+4 y, u=\phi(\hat{z}, y)$ solves the stabilization problem.

\subsubsection{Design from the natural stability margin.}

We are unaware of any design from the natural stability margin with a dynamic error model analysis. Actually, we don't know even if this makes sense since the system from which we would like to exploit the natural stability margin is the $w$ or $(w, y)$-subsystem which is to be designed.

\subsection{Cancellation.}

The only result we have been able to re-interpret along the lines of an indirect approach with a

\footnotetext{
6 Achieving this $L^{1}$ integrability may be difficult in general. It may be useful to modify the observer as suggested in (Arcak, 2005).
} 
cancellation design concerns systems for which we can find coordinates so that the dynamic is linear in the unmeasured state components. In this case (18) takes the form:

$\dot{\chi}=A(u, y) \chi+B(u, y), \dot{y}=C(u, y)^{T} \chi+d(u, y)$. and the following result holds.

Proposition 6. (Praly, 1992) If:

(1) there exists a CLF $U$, with bounded gradient and Hessian, associated to a state feedback $\phi$;

(2) there exist a function $(u, y) \mapsto\left(K_{\chi}, K_{y}\right)$ and and a positive definite symmetric matrix $P$ satisfying, for all $(u, y)$,

$Q(u, y)=\operatorname{sym}\left(P\left(\begin{array}{cc}A(u, y) & -K_{\chi}(u, y) \\ C(u, y)^{T} & -K_{y}(u, y)\end{array}\right)\right)<0 ;$

then we can solve the output feedback stabilization problem.

As the gradient of $U$ is bounded, there exists a real number $c$ such that the function $V$, defined as:

$$
\begin{array}{r}
V((\chi, y),(\hat{\chi}, \hat{y}))=U(\hat{\chi}, y)-\frac{\partial U}{\partial \chi}(\hat{\chi}, y)[\hat{\chi}-\chi] \\
+c\left([\hat{\chi}-\chi]^{T}[\hat{y}-y]\right) P\left(\begin{array}{c}
\hat{\chi}-\chi \\
\hat{y}-y
\end{array}\right),
\end{array}
$$

is lower bounded, with the origin as unique critical point, and is radially unbounded. Then, by using the fact that the Hessian of $U$ is bounded, we can design $\left(v_{\chi}, v_{y}\right)$ in the system:

$$
\begin{aligned}
\dot{\hat{\chi}} & =A(u, y) \hat{\chi}+B(u, y)+K_{\chi}(u, y)[y-\hat{y}]+v_{\chi}, \\
\dot{\hat{y}} & =C(u, y)^{T} \hat{\chi}+d(u, y)+K_{y}(u, y)[y-\hat{y}]+v_{y}, \\
u & =\phi(\hat{\chi}, y),
\end{aligned}
$$

to obtain an output feedback guaranteeing:

$$
\begin{aligned}
\dot{V}((\chi, y), & (\hat{\chi}, \hat{y})) \leq \dot{U}_{\text {nom }}(\hat{\chi}, y) \\
& +c\left([\hat{\chi}-\chi]^{T}[\hat{y}-y]\right) Q(u, y)\left(\begin{array}{c}
\hat{\chi}-\chi \\
\hat{y}-y
\end{array}\right) .
\end{aligned}
$$

The key point here is the modification of the nominal observer by $\left(v_{\chi}, v_{y}\right)$ to realize the cancellation. Such an observer modification is also present in (Kanellakopoulos et al., 1992; Arcak, 2005).

\section{DOMINATION VIA A DOMINANT MODEL.}

Up to now, both for the direct and indirect case, we have presented the domination design as exploiting the negativeness of $\dot{U}_{\text {nom }}$ obtained for a CLF $U$ for the nominal system:

$$
\dot{x}=f(x, u) \text {. }
$$

We can push this strategy further by working only with a "dominant" approximation of this nominal system. In the linear case, the archetype of such an approach says that, by designing a high gain linear output feedback for the chain of integrators $\dot{y}_{1}=y_{2}, \ldots, \dot{y}_{n_{y}-1}=y_{n_{y}}, \dot{y}_{n_{y}}=u$,

and by adjusting the gain, we can solve the stabilization problem by output feedback for any minimum phase linear system with relative degree $n_{y}$.

A way to extend the above result is to preserve the linear structure linked to a vector space but now with scalars which are no more real numbers but functions of the output. In this case, the chain of integrators is:

$$
\begin{aligned}
\dot{y}_{1} & =a_{1}\left(y_{1}\right) y_{2}, \\
& \vdots \\
\dot{y}_{n_{y}-1} & =a_{n_{y}-1}\left(y_{1}\right) y_{n_{y}}, \\
\dot{y}_{n_{y}} & =a_{n_{y}}\left(y_{1}\right) u .
\end{aligned}
$$

For this system, we design again a high gain linear output feedback, with linearity taken in the new sense. However, because the scalars vary along the solutions, the high gain has to be dynamical. This makes the analysis much more involved. Following this route with an indirect approach, the following result can be obtained.

Proposition 7. (Krishnamurthy and Khorrami, $2004)^{7}$. Consider the system:

$$
\begin{aligned}
\dot{y}_{1} & =a_{1}(y) y_{2}+\delta_{1}, \\
& \vdots \\
\dot{y}_{n_{y}-1} & =a_{n_{y}-1}(y) y_{n_{y}}+\delta_{n_{y}-1}, \\
\dot{y}_{n_{y}} & =a_{n_{y}}(y) u+\delta_{n_{y}}, \\
y & =y_{1} .
\end{aligned}
$$

If:

(1) the functions $a_{i}$ satisfy, for some real numbers $c$ and $C$ :

$0<c \leq a_{1}(y) \quad, \quad c \leq \frac{a_{i}(y)}{a_{1}(y)} \leq C \quad \forall y ;$

(2) $\delta_{i}$ is the output of an ISS system with $\left(y_{1}, . ., y_{i}\right)$ as input with a linear (in the new sense) input-output gain, i.e. there exists a function $\mu_{i}$ such that we have ${ }^{8}$ :

$$
\left|\delta_{i}\right| \leq \sqrt{\mu_{i}\left(y_{1}\right) V_{i}}+\mu_{i}\left(y_{1}\right)\left[\sum_{j=1}^{i}\left|y_{j}\right|\right]
$$

where, along each solution of the system, $V_{i}$ satisfies the differential inequality:

$$
\frac{\dot{V}_{i}}{\alpha_{i}} \leq-\left(V_{i}-\mu_{i}\left(y_{1}\right)\left[\sum_{j=1}^{i}\left|y_{j}\right|\right]^{2}\right)
$$

\footnotetext{
7 In (Krishnamurthy and Khorrami, 2004, Theorem 3) a more general result is established. However it is not an exact solution to our stabilization problem, since part of the state of the controller does not converge to a prescribed value.

8 The summation from $j=1$ to $j=i$ says that we are dealing with a system in feedback form.
} 
with $\alpha_{i}>0$

then we can solve the output feedback stabilization problem.

A counterpart of Proposition 7 for a system in feedforward form, i.e. with a summation from $j=i+2$ to $j=n_{y}$, is given in (Krishnamurthy and Khorrami, 2005).

Another extension exploits a generalization of the natural homogeneity property linked to a linear system. Precisely, we observe that, if we change $u, y_{i}$ and the time $t$ as:

$$
\mathcal{u}=\lambda^{1+d} u, \quad \mathcal{y}_{i}=\lambda^{1-\left(n_{y}-i\right) d} y_{i}, \quad \mathcal{T}=\lambda^{-d} t,
$$

then the chain of integrators (22) becomes:

$\frac{d y_{1}}{d \mathcal{T}}=\mathcal{y}_{2}, \ldots, \frac{d \mathcal{y}_{n_{y}-1}}{d \mathcal{T}}=\mathcal{y}_{n_{y}}, \frac{d \mathcal{y}_{n_{y}}}{d \mathcal{T}}=\mathcal{u}$, which has exactly the same form. This is called invariance by weighted homogeneity. Systems exhibiting such an invariance have properties extremely interesting in the stabilization context. To take advantage of these properties the idea is to design the output feedback so that the "dominant" part of the closed loop system exhibits this invariance. This technique, exploited with an indirect approach, leads to the following result.

Proposition 8. (Andrieu et al, 2006) Consider the system:

$$
\begin{aligned}
\dot{y}_{1} & =y_{2}+\delta_{1}, \\
& \vdots \\
\dot{y}_{n_{y}-1} & =y_{n_{y}}+\delta_{n_{y}-1}, \\
\dot{y}_{n_{y}} & =u+\delta_{n_{y}}, \\
y & =y_{1} .
\end{aligned}
$$

If $\delta_{i}$ is the output of an ISS system with $\left(y_{1}, \ldots, y_{i}\right)$ as input such that there exist two positive real numbers $d_{0}$ and $d_{\infty}$ satisfying $-1<$ $d_{0} \leq d_{\infty}<\frac{1}{n_{y}-1}$ and a positive real number $\mu$ such that we have:

$$
\begin{aligned}
\left|\delta_{i}\right| \leq & \sqrt{\mu V_{i}} \\
& +\mu\left[\sum_{j=1}^{i}\left|y_{j}\right|^{\frac{1-d_{0}\left(n_{y}-i-1\right)}{1-d_{0}\left(n_{y}-j\right)}}+\left|y_{j}\right|^{\frac{1-d_{\infty}\left(n_{y}-i-1\right)}{1-d_{\infty}\left(n_{y}-j\right)}}\right]
\end{aligned}
$$

where, along each solution of the system, $V_{i}$ satisfies the differential inequality:

$$
\begin{aligned}
\frac{\dot{V}_{i}}{\alpha_{i}} & \leq-\left(V_{i}-\right. \\
\mu & {\left.\left[\sum_{j=1}^{i}\left|y_{j}\right|^{\frac{1-d_{0}\left(n_{y}-i-1\right)}{1-d_{0}\left(n_{y}-j\right)}}+\left|y_{j}\right|^{\frac{1-d_{\infty}\left(n_{y}-i-1\right)}{1-d_{\infty}\left(n_{y}-j\right)}}\right]^{2}\right), }
\end{aligned}
$$

with $\alpha_{i}>0$, then we can solve the output feedback stabilization problem.
A counterpart of Proposition 8 for a system in feedforward form, i.e. with a summation from $j=i+2$ to $j=n_{y}$, is given in (Andrieu et al, 2006).

By developing further the formalism of weighted homogeneity, we can deal with systems which can be represented by:

$$
\begin{aligned}
\dot{y}_{1} & =\operatorname{sign}\left(y_{1}\right)\left|y_{2}\right|^{p_{1}}+\delta_{1}, \\
& \vdots \\
\dot{y}_{n_{y}-1} & =\operatorname{sign}\left(y_{n_{y}}\right)\left|y_{n_{y}}\right|^{p_{n_{y}-1}}+\delta_{n_{y}-1}, \\
\dot{y}_{n_{y}} & =u+\delta_{n_{y}}, \\
y & =y_{1},
\end{aligned}
$$

where the $p_{i}$ are real numbers larger or equal to 1 . The following result can be obtained with a direct approach.

Proposition 9. (Polendo and Qian, 2005) (See also (Qian, 2005)). Consider system (23). If there exist a positive real number $d$ and a positive real number $\mu$ such that we have:

where:

$$
\left|\delta_{i}\right| \leq \mu\left[\sum_{j=1}^{i}\left|y_{j}\right|^{\frac{r_{i}+d}{r_{j}}}\right]
$$

$$
r_{1}=1 \quad, \quad r_{i}+d=r_{i+1} p_{i},
$$

then we can solve the output feedback stabilization problem.

\section{CONCLUDING REMARKS.}

A lot of effort has been devoted to the output feedback stabilization problem. But there are still many open problems and the need to clarify and to develop further the theory. In this regard, we have proposed a framework for studying, in a unified way, globally stabilizing output feedback designs. The core is a classification based on a two by two matrix (see Table 1) with direct/indirect as entries in one direction and domination/cancellation in the other, where:

- Direct means that the design is done to directly address the stabilization problem whereas indirect says that this problem is solved only because some kind of observer converges.

- Domination means that stronger terms are used to dominates the bad ones, whereas cancellation means that these bad terms are simply eliminated.

We have seen that the domination approach can be developed further to be applicable, not to the given system, but only to a "dominant" approximation. It is even possible to combine direct and indirect techniques in a domination design. This is done for instance in (Karagiannis et al., 2005) to deal with systems in normal form (2). 
By far, the indirect/domination approach is the most frequently exploited in the theoretical contributions. Instead the direct approach is likely to be the most frequently used in applications. In particular the direct/cancellation version is very appealing since it exploits even more the specificity of the system as opposed to domination designs which ignore it, at least in part.

In the domination designs, we need state feedbacks able to enforce robustness properties to the stability. This has motivated many specific contributions, see (Andrieu and Praly, 2006; Freeman and Kokotović, 1993; Kanellakopoulos et al., 1991; Krishnamurthy and Khorrami, 2006; Jiang et al., 2004; Lin and Qian, 2000) for instance.

Also, the need of observers is apparent, be for only a function of the state (a reparameterized version of the state feedback) or the state itself. To answer this need new observers going significantly beyond the linear paradigm have been proposed. See (Arcak and Kokotović, 2001-TAC; Andrieu et al, 2006; Krishnamurthy and Khorrami, 2006; Praly, 2003; Praly and Astolfi, 2005; Qian and Lin, 2006) for instance.

\section{REFERENCES}

A. Ailon, R. Ortega, An observer-based set-point controller for robot manipulators with flexible joints, Systems \& Control Letters 21 (1993) 329-335

V. Andrieu, Bouclage de sortie et observateur. Thèse de doctorat de l'École des Mines de Paris, Spécialité: Mathématiques et Automatique. Décembre 2005.

V. Andrieu, L. Praly, Global asymptotic stabilization for non-minimum phase non linear systems admitting a strict normal form. Submitted publication. September 2006. See also: Proceedings of the 44th IEEE Conference on Decision and Control, December 2005.

V. Andrieu, L. Praly, A. Astolfi, Homogeneous approximation, recursive observer design and output feedback. Submitted publication. November 2006 See also: Nonlinear output feedback design via domination and generalized weighted homogeneity. Proceedings of the 45th IEEE Conference on Decision and Control, December 2006.

M. Arcak, Certainty equivalence output feedback design with circle criterion observers. IEEE Transactions on Automatic Control, Vol. 50, No. 6, June 2005.

M. Arcak, P. Kokotović, Observer-Based Control of Systems with Slope-Restricted Nonlinearities. IEEE Transactions on Automatic Control, Vol. 46, No. 7, July 2001.
M. Arcak, P. Kokotović, Nonlinear observers: a circle criterion design and robustness analysis. Automatica 37 (2001) 1923-1930.

A. N. Atassi and H. K. Khalil, A separation principle for the stabilization of a class of nonlinear systems, IEEE Transactions on Automatic Control, Vol. 44, No. 9, September 1999.

C. Byrnes, A. Isidori, Asymptotic stabilization of minimum phase nonlinear systems. IEEE Transactions on Automatic Control, Vol. 36, No. 10, October 1991.

N. Chung, Input to state stability with respect to measurement disturbances for one dimensional systems. ESAIM: Control, Optimisation and Calculus of Variations, April 1999, Vol. 4, p. 99-121

J.-M. Coron, On the stabilization of controllable and observable systems by output feedback law, Mathematics of Control, Signals and Systems (1994) 7: 187-216.

R. Freeman, Global internal stabilizability does not imply global external stabilizability for small sensor disturbances. IEEE Transactions on Automatic Control, Vol. 40, No. 12, December 1995

R. Freeman, P. Kokotović, Global robustness of nonlinear systems to state measurement disturbances. Proceedings of the 32nd IEEE Conference on Decision and Control, December 1993.

R. Freeman, P. Kokotović, Tracking controllers for systems linear in the unmeasured states, Automatica 32 (1996) 735-746

P. Ioannou, J. Sun, Robust adaptive control. Prentice Hall, 1996.

Z. P. Jiang, I. Mareels, D.J. Hill, J. Huang, A unifying framework for global regulation via nonlinear output feedback: from ISS to iISS, IEEE Transactions on Automatic Control, Vol. 49, No. 4, April 2004.

I. Kanellakopoulos, P. Kokotović, A. Morse. Adaptive Output-Feedback Control of a class of Nonlinear Systems. Proceedings of the 30th IEEE Conference on Decision and Control, December 1991.

I. Kanellakopoulos, M. Krstić, P. Kokotović, Interlaced controller-observer design for adaptive nonlinear control. Proceedings of the of the 1992 American Control Conference, June 1992.

D. Karagiannis, Z-P. Jiang, R. Ortega, A. Astolfi, Output-feedback stabilization of a class of uncertain non-minimum-phase nonlinear systems. Automatica 41 (2005) 1609-1615,

P. Kokotović, The joy of feedback: nonlinear and adaptive, IEEE Control Systems Magazine, Vol. 12, No. 3, June 1992.

P. Krishnamurthy, F. Khorrami, and Z. P. Jiang, Global Output Feedback Tracking for Nonlin- 
ear Systems in Generalized Output-Feedback Canonical Form. IEEE Transactions on Automatic Control, Vol. 47, No. 5, May 2002.

P. Krishnamurthy, F. Khorrami, Dynamic highgain scaling: state and output feedback with application to systems with ISS appended dynamics driven by all states. IEEE Transactions on Automatic Control, Vol. 49, No. 12, December 2004.

P. Krishnamurthy, F. Khorrami, Adaptive outputfeedback stabilization and disturbance attenuation for feedforward systems with ISS appended dynamics. Proceedings of the 44th IEEE Conference on Decision and Control, and the European Control Conference 2005, December 2005.

P. Krishnamurthy, F. Khorrami, On uniform solvability of parameter-dependent Lyapunov inequalities and applications to various problems. SIAM J. Control Optim. Vol. 45, No. 4, pp. 1147-1164.

W. Lin, C Qian, Adding one power integrator: a tool for global stabilization of high-order lower-triangular systems. Systems $\&$ Control Letters 39 (2000) 339-354

R. Marino, P. Tomei, Global adaptive outputfeedback control of nonlinear systems. Proceedings of the 30th IEEE Conference on Decision and Control, December 1991.

R. Marino, P. Tomei, A class of globally output feedback stabilizable nonlinear nonminimum phase systems. IEEE Transactions on Automatic Control, Vol. 50, No. 12, December 2005.

F. Mazenc, L. Praly, Global stabilization for nonlinear systems. Rapport interne CAS. January 1993

F. Mazenc, L. Praly, W. P. Dayawansa, Global stabilization by output feedback: Examples and Counter-Examples, Systems $\&$ Control Letters 23 (1994) 119-125

R. Ortega, A. Loria, R. Kelly, L. Praly, On passivity-based output feedback global stabilization of Euler-Lagrange systems, Int. J. of Robust and Nonlinear Control, Vol. 5, 313323, 1995.

Z. Pan, K. Ezal, A. Krener, P. Kokotović, Backstepping design with local optimality matching. IEEE Transactions on Automatic Control, Vol. 46, No. 7, July 2001

J. Polendo, C. Qian, A Generalized Framework for Global Output Feedback Stabilization of Genuinely Nonlinear Systems. Proceedings of the 44th IEEE Conference on Decision and Control, and the European Control Conference 2005, December 2005.

J.-B. Pomet, Sur la commande adaptative des systèmes non linéaires. Thèse de doctorat de l'École des Mines de Paris, Spécialité:
Mathématiques et Automatique. Septembre 1989.

J.-B. Pomet, R. Hirschorn, W. Cebuhar, Dynamic output feedback regulation for a class of nonlinear systems. Mathematics of Control, Signals and Systems (1993) 6: 106-124.

L. Praly, Asymptotic stabilization via output feedback for lower triangular systems with output dependent incremental rate. IEEE Transactions on Automatic Control, Vol. 48, No. 6, June 2003.

L. Praly, Lyapunov design of a dynamic output feedback for systems linear in their unmeasured state components. Proceedings of the 2nd IFAC Symposium on Nonlinear Control Systems (NOLCOS 1992). June 1992.

L. Praly, M. Arcak, A Sufficient Condition for Stability of Nonlinear Observer-Based Controllers. Systems $\&$ Control Letters 53 (2004) 311-320

L. Praly, A. Astolfi, Global asymptotic stabilization by output feedback under a state norm detectability assumption. Proceedings of the 44th IEEE Conference on Decision and Control, December 2005

C. Prieur, L. Praly, A tentative direct Lyapunov design of output feedbacks. Proceedings of the 6th IFAC Symposium on Nonlinear Control Systems (NOLCOS 2004). September 2004.

C. Qian, A Homogeneous Domination Approach for Global Output Feedback Stabilization of a Class of Nonlinear Systems, Proceedings of the 2005 American Control Conference Page(s):4708 - 4715 vol. 7 June 2005

C. Qian, W. Lin, Recursive observer design, homogeneous approximation, and nonsmooth output feedback stabilization of nonlinear systems, IEEE Transactions on Automatic Control, Vol. 51, No. 9, September 2006.

H. Shim, A. Teel, Asymptotic controllability and observability imply semi-global practical asymptotic stabilizability by sampled-data output feedback. Automatica 39 (2003) 441454.

E. Sontag, Further facts about input to state stabilization IEEE Transactions on Automatic Control, Vol. 35, No. 4, April 1990

E. Sontag, Input to state stability: Basic concepts and results. In P. Nistri and G. Stefani, editors, Nonlinear and Optimal Control Theory, pages 163-220. Springer-Verlag, Berlin, 2006

A. Teel, L. Praly, Global stabilizability and observability imply semi-global stabilizability by output feedback, Systems \& Control Letters 22 (1994) 313-325 